\title{
NOTE ON KKM MAPS AND APPLICATIONS
}

\author{
Y. Q. CHEN, Y. J. CHO, J. K. KIM, AND B. S. LEE \\ Received 6 March 2005; Revised 20 July 2005; Accepted 11 August 2005
}

We apply the KKM technique to study fixed point theory, minimax inequality and coincidence theorem. Some new results on Fan-Browder fixed point theorem, Fan's minimax theorem and coincidence theorem are obtained.

Copyright (c) 2006 Y. Q. Chen et al. This is an open access article distributed under the Creative Commons Attribution License, which permits unrestricted use, distribution, and reproduction in any medium, provided the original work is properly cited.

\section{Introduction}

In 1929, the KKM map was introduced by Knaster et al. [13] and it provides the foundation for many well-known existence results, such as Ky Fan's minimax inequality theorem, Ky Fan-Browder's fixed point theorem, Nash's equilibrium theorem, HartmanStampacchia's variational inequality theorem and many others (see [1, 2, 5-12, 14-17]). The central idea of applying KKM theory to prove that a family of sets has nonempty intersection is to find a suitable space and a mapping defined on that space such that this mapping is a KKM mapping and the original family of sets has finite intersection property provided the resulted family of sets by this mapping has finite intersection property. Based this idea, we first introduce a large class of mappings that can be interpreted as KKM mappings, then we apply the KKM technique to study fixed point theory, minimax inequality and coincidence theorem. A new concept on lower (upper) semi-continuous function is given and some new results on Fan-Browder's fixed point theorem, Fan's minimax theorem and coincidence theorem are obtained.

\section{The KKM maps}

In the sequel, let $X$ be a set and $2^{X}$ be the collection of nonempty subsets of $X$. To begin our results, let us first recall the following definition.

Definition 2.1. Let $E$ be a subset of topological vector space $X$. A map $G: E \rightarrow 2^{X}$ is called a KKM map if 


$$
\operatorname{co}\left\{x_{1}, x_{2}, \ldots, x_{n}\right\} \subseteq \bigcup_{i=1}^{n} G\left(x_{i}\right)
$$

for $x_{i} \in E, i=1,2, \ldots, n$.

Definition 2.2. Let $E$ be a set and $X$ be a topological space. A map $G: E \rightarrow 2^{X}$ is called a map with the KKM property if there exists a topological vector space $Y$ such that, for any $\left\{x_{i}: 1 \leq i \leq n\right\} \subseteq E$, there exist $F=\left\{y_{i}: 1 \leq i \leq n\right\} \subseteq Y$, a closed (or closed under appropriate topology) mapping $L: X \rightarrow Y$ or $2^{Y}$, that is, maps closed set to closed set, and $G^{\prime}: F \rightarrow 2^{X}$ with $G^{\prime}\left(y_{i}\right) \subseteq G\left(x_{i}\right)$ for $i=1,2, \ldots, n$ such that the composition mapping $L G^{\prime}$ : $F \rightarrow 2^{Y}$ defined by $L G^{\prime}(f)=\cup_{x \in G^{\prime}(f)} L(x)$ for $f \in F$ is a $K K M$ map and $\cap_{i=1}^{n} L G^{\prime}\left(y_{i}\right) \neq \varnothing$ implies that $\cap_{i=1}^{n} G\left(x_{i}\right) \neq \varnothing$.

Remark 2.3. Definition 2.2 simply says that the map $G$ has the KKM property if $G$ or the part of $G$ can be mapped onto another space such that the composite map is a KKM map. One can easily check that the generalized KKM map in $[4,18]$ is a map with the KKM property.

In the following, we give some examples of maps with the KKM property.

Example 2.4. Let $E=[0,1]$ be the closed interval of $R, X=R$, and let $G: E \rightarrow 2^{X}$ be a map with $G(x)=(1,2+x)$ for $x \in E$. For any $\left(x_{i}\right) \subset[0,1], i=1,2, \ldots, n$, put $y_{i}=3 / 2+x_{i}$, $F=\left\{y_{1}, y_{2}, \ldots, y_{n}\right\}, Y=R$, and define $G^{\prime}: F \rightarrow 2^{Y}$ by $G^{\prime}\left(y_{i}\right)=\left[3 / 2,7 / 4+x_{i}\right]$. Take $L$ as the identity mapping on $R$. Then the map $L G^{\prime}=G^{\prime}$ is a $K K M$ map and so $G$ is a map with the KKM property.

Example 2.5. Let $\phi:[0, \infty) \rightarrow R$ be the convex function defined by

$$
\phi(x)= \begin{cases}1 & \text { if } x=0 \\ (x-1)^{2}-1 & \text { if } x>0\end{cases}
$$

Define $G:[0, \infty) \rightarrow 2^{R}$ by $G(x)=\{y: \phi(y) \leq \phi(x)\}$. It is easy to see that $\phi$ is not lower semi-continuous at 0 and so $G(2)=\{y: \phi(y) \leq \phi(2)\}$ is not closed. For $\left\{x_{i}: 1 \leq i \leq\right.$ $n\} \subset[0, \infty)$, if $\phi\left(x_{i}\right)<0$ or $\phi\left(x_{i}\right) \geq 1$, we set $y_{i}=x_{i}$, otherwise, set $y_{i}=x_{i} / 2$. Put $F=$ $\left\{y_{1}, y_{2}, \ldots, y_{n}\right\}, X=Y=R$, and define $G: F \rightarrow 2^{X}$ by $G^{\prime}\left(y_{i}\right)=\left\{y: \phi(y) \leq \phi\left(y_{i}\right)\right\}$. Take $L$ as the identity mapping on $R$. Then $L G^{\prime}=G^{\prime}$ is a KKM map on $F=\left\{y_{i}: 1 \leq i \leq n\right\}$, thus $G$ is a map with the KKM property.

The following results are direct consequences of the KKM theorem.

Theorem 2.6. Let $X$ be a topological space and $E$ be a set. Suppose that $G: E \rightarrow 2^{X}$ is a closed valued map with the KKM property. Then $\{G(x)\}_{x \in E}$ has a finite intersection property.

Theorem 2.7 (Ky Fan's theorem). Let $X$ be a topological space and $E$ be a subset of $X$. If $G: E \rightarrow 2^{X}$ is a closed valued map with the KKM property and there is a set $G(x)$ such that $G(x)$ is compact. Then $\cap_{x \in E} G(x) \neq \varnothing$. 
Y. Q. Chen et al. 3

\section{Fan-Browder's fixed point theorem without compactness condition}

The following result is a generalization of Fan-Browder's fixed point theorem without compactness condition.

Theorem 3.1 (Fan-Browder's fixed point theorem). Let $E$ be a convex subset of a vector space $X$ and $G: E \rightarrow 2^{E}$ be a map satisfying the following conditions:

(1) there exists $\left\{y_{i}: 1 \leq i \leq n\right\} \subset E$ such that $\operatorname{co}\left\{y_{i}: 1 \leq i \leq n\right\} \subseteq \cup_{i=1}^{n} G^{-1}\left(y_{i}\right)$ and $G^{-1}\left(y_{i}\right) \cap \operatorname{co}\left\{y_{i}: 1 \leq i \leq n\right\}$ is open in $\operatorname{co}\left\{y_{i}: 1 \leq i \leq n\right\}$ with $\operatorname{co}\left\{y_{i}: 1 \leq i \leq n\right\}$ inherited with the Euclidean topology, where $G^{-1}(y)=\{x \in E: y \in G(x)\}$;

(2) $G(y)$ is convex for all $y \in E$.

Then $G$ has a fixed point.

Proof. Let $F=\left\{y_{i}: 1 \leq i \leq n\right\}$. Define a map $K: F \rightarrow 2^{\text {co } F}$ by

$$
K\left(y_{i}\right)=\operatorname{co} F \backslash G^{-1}\left(y_{i}\right) \bigcap \operatorname{co} F
$$

for $i=1,2, \ldots, n$. We may assume that $K\left(y_{i}\right) \neq \varnothing$ for $i=1,2, \ldots$. (Otherwise, $K\left(y_{i}\right)=\varnothing$ for some $i$ and so we have $\operatorname{co} F \subset G^{-1}\left(y_{i}\right)$. Thus $y_{i}$ is a fixed point of $G$, and the conclusion holds.) One can easily see that

$$
\bigcap_{i=1}^{n} K\left(y_{i}\right)=\operatorname{co} F \backslash \bigcup_{i=1}^{n} G^{-1}\left(y_{i}\right) \bigcap \operatorname{co} F .
$$

By assumption (1), we have $\cap_{i=1}^{n} K\left(y_{i}\right)=\varnothing$. In view of Theorem 2.6, $K$ cannot be a KKM map on $\left\{y_{i}: 1 \leq i \leq n\right\}$. Hence there exist $y_{i_{1}}, y_{i_{2}}, \ldots, y_{i_{k}}$ such that $\operatorname{co}\left\{y_{i_{1}}, y_{i_{2}}, \ldots, y_{i_{k}}\right\} \nsubseteq$ $\cup_{j=1}^{k} K\left(y_{i_{j}}\right)$, that is, there exists $y \in \operatorname{co}\left\{y_{i_{1}}, y_{i_{2}}, \ldots, y_{i_{k}}\right\}$ such that $y \notin K\left(y_{i_{j}}\right)$ for $j=1,2, \ldots$, $k$. Thus we have

$$
y \in G^{-1}\left(y_{i_{j}}\right), \quad j=1,2, \ldots, k,
$$

that is, $y_{i_{j}} \in G(y)$ for $j=1,2, \ldots, k$ and the convexity of $G(y)$ immediately implies that $y \in G(y)$. This completes the proof.

Remark 3.2. Theorem 3.1 only requires the intersection $G^{-1}(y) \cap \operatorname{co} F$ for $y \in F$ is relatively open in the convex hull of some finite subset $F$ of $E$ and also $E$ is not compact, which is different to the result in [3]. See also Theorem 1.2 on page 143 of Granas-Dugundji's book [11].

Example 3.3. Let $E=(0,1)$ and a map $T: E \rightarrow 2^{E}$ be defined by

$$
T x= \begin{cases}\left(x, x+\frac{1}{2}\right) & \text { if } x \in\left(0, \frac{1}{2}\right), \\ \left(\frac{1}{3}, x+\frac{1}{4}\right) & \text { if } x \in\left[\frac{1}{2}, \frac{3}{4}\right] \\ \left(x-\frac{1}{2}, x\right) & \text { otherwise. }\end{cases}
$$


It is obvious that $E$ is not compact and $T x$ is convex for all $x \in E$. Put $y_{1}=1 / 2$ and $y_{2}=3 / 4$. Then it follows that

$$
\begin{gathered}
\operatorname{co}\left\{y_{1}, y_{2}\right\}=\left[\frac{1}{2}, \frac{3}{4}\right] \subset T^{-1} y_{1} \bigcup T^{-1} y_{2}, \\
T^{-1} \frac{1}{2} \bigcap\left[\frac{1}{2}, \frac{3}{4}\right]=\left[\frac{1}{2}, \frac{3}{4}\right], \\
T^{-1} \frac{3}{4} \bigcap\left[\frac{1}{2}, \frac{3}{4}\right]=\left(\frac{1}{2}, \frac{3}{4}\right]
\end{gathered}
$$

are open in $[1 / 2,3 / 4]$. Therefore, the map $T$ satisfies the conditions of Theorem 2.6.

Corollary 3.4. Let $C$ be a nonempty convex subset of a topological vector space $E$ and $V$ be an open convex subset with $0 \in V$. Suppose that a map $T: C \rightarrow E$ is continuous and $T(C) \subset \cup_{i=1}^{n}\left\{y_{i}+V\right\}$, where $y_{i} \in C$ for $i=1,2, \ldots, n$. Then there exists $x_{0} \in C$ such that $T x_{0} \in x_{0}+V$.

Proof. Let a map $G: C \rightarrow 2^{C}$ be defined by

$$
G(x)=\{y \in C: T x-y \in V\} .
$$

Then $G(x)$ is convex for all $x \in C$ since $V$ is convex. The continuity of $T$ implies that $G^{-1}\left(y_{i}\right)$ is open. Moreover, $C=\cup_{i=1}^{n} G^{-1}\left(y_{i}\right)$ and thus

$$
\operatorname{co}\left\{y_{1}, y_{2}, \ldots, y_{n}\right\} \subseteq \bigcup_{i=1}^{n} G^{-1}\left(y_{i}\right) .
$$

Therefore, by Theorem 3.1, we know that there exists $x_{0} \in C$ such that $x_{0} \in G\left(x_{0}\right)$. This implies that $T x_{0} \in x_{0}+V$.

Corollary 3.5. Let $C$ be a nonempty convex subset of a locally convex space $E$ and $K$ be a convex compact subset of E. Suppose that $T: C \rightarrow E$ is continuous and $T(C) \subset \cup_{i=1}^{n}\left\{y_{i}+K\right\}$, where $y_{i} \in C$ for $i=1,2, \ldots, n$. Then there is an $x_{0} \in C$ such that $T x_{0} \in x_{0}+K$.

\section{Coincidence theorem and minimax theorem}

Theorem 4.1 (Ky Fan's coincidence theorem). Let $X$ and $Y$ be nonempty convex subsets of topological vector spaces $E$ and $F$, respectively. Let $A, B: X \rightarrow 2^{Y}$ be two maps satisfying the following conditions:

(1) there exists $x_{i} \in X$ such that $A x_{i}$ is open for $i=1,2, \ldots, n, Y=\cup_{i=1}^{n} A x_{i}$ and $A^{-1} y$ is a convex set for each $y \in Y$;

(2) there exists $y_{j} \in y$ such that $B^{-1} y_{j}$ is open for $j=1,2, \ldots, m, X=\cup_{j=1}^{m} B^{-1} y_{j}$ and $B x$ is a convex set for each $x \in Y$.

Then there exists $x_{0} \in X$ such that $A x_{0} \cap B x_{0} \neq \varnothing$.

Proof. Let a map $K: X \times Y \rightarrow 2^{X \times Y}$ be defined by

$$
K(x, y)=X \times Y \backslash\left(B^{-1} y \times A x\right)
$$


for all $(x, y) \in X \times Y$. By the assumptions, we have

$$
X \times Y=\bigcup_{i=1}^{n} \bigcup_{j=1}^{m}\left(B^{-1} y_{j} \times A x_{i}\right) \text {. }
$$

Therefore, we have

$$
\bigcap_{i=1}^{n} \bigcap_{j=1}^{m} K\left(x_{i}, y_{j}\right)=\varnothing .
$$

In view of Theorem 2.6, we know that $K$ cannot be a KKM map on $\left\{x_{i}: 1 \leq i \leq n\right\} \times\left\{y_{j}\right.$ : $1 \leq j \leq m\}$. So there exist $x_{0}, x_{i_{1}}, x_{i_{2}}, \ldots, x_{i_{l}}$ and $y_{0}, y_{j_{1}}, y_{j_{2}}, \ldots, y_{j_{k}}$ such that $x_{0} \in \operatorname{co}\left\{x_{i_{1}}, x_{i_{2}}\right.$, $\left.\ldots, x_{i_{l}}\right\}, y_{0} \in \operatorname{co}\left\{y_{j_{1}}, y_{j_{2}}, \ldots, y_{j_{k}}\right\}$ and

$$
\left(x_{0}, y_{0}\right) \notin \bigcup_{s=1}^{l} \bigcup_{t=1}^{k} K\left(x_{i_{s}}, y_{j_{t}}\right),
$$

which implies that

$$
\left(x_{0}, y_{0}\right) \in\left(B^{-1} y_{j_{t}} \times A x_{i_{s}}\right)
$$

for $s=1, \ldots, l$ and $t=1,2, \ldots, k$. By the convexities of $A^{-1} x$ and $B y$, we have $y_{0} \in A x_{0}$ and $y_{0} \in B x_{0}$. This completes the proof.

Remark 4.2. The classical Ky Fan's coincidence theorem assume that both $X$ and $Y$ are compact. See Theorem 3.12 in Singh-Watson-Srivastava's book [15]. We do not require this condition in Theorem 4.1 .

Definition 4.3. Let $X$ be a topological space. A function $f: X \rightarrow R$ is said to be lower semicontinuous from above at $x_{0}$ if, for any net $\left(x_{t}\right)_{t \in T}$ with $x_{t} \rightarrow x_{0}, f\left(x_{t^{\prime}}\right) \leq f\left(x_{t}\right)$ for $t^{\prime} \geq t$ implies that $f\left(x_{0}\right) \leq \lim _{t} f\left(x_{t}\right)$. Similarly, $f$ is said to upper semi-continuous from below at $x_{0}$ if, for any net $\left(x_{t}\right)_{t \in T}$ with $x_{t} \rightarrow x_{0}, f\left(x_{t}\right) \leq f\left(x_{t^{\prime}}\right)$ for $t \leq t^{\prime}$ implies that $f\left(x_{0}\right) \leq$ $\lim _{t} f\left(x_{t}\right)$.

One can easily see that a lower (resp., upper) semi-continuous function is also a lower (resp., upper) semi-continuous from above (resp., below) function.

The following example shows that the converse is not true.

Example 4.4. Let a function $f: R \rightarrow R$ be defined by

$$
f(x)= \begin{cases}x+1 & \text { if } x \geq 0 \\ x & \text { if } x<0\end{cases}
$$

Since $R$ is a metric space, we consider a sequence $\left\{x_{n}\right\}$ such that $x_{n} \rightarrow 0$ with $f\left(x_{1}\right) \geq$ $f\left(x_{2}\right) \geq \cdots \geq f\left(x_{n}\right) \geq \cdots$. Then, by the definition of $f(x)$, we know that $x_{n} \geq 0$ for all $n \geq 1$. Therefore, it follows that

$$
\lim _{n \rightarrow \infty} f\left(x_{n}\right)=1=f(0)
$$


and so $f$ is lower semi-continuous from above at 0 . If we take $x_{n}=-1 / n$, then we have

$$
\lim _{n \rightarrow \infty} f\left(x_{n}\right)=0<f(0)
$$

and so $f$ cannot be lower semi-continuous at 0 .

Lemma 4.5. Let $X$ be a compact topological space and $f: X \rightarrow R$ be a real valued function. If $f$ is lower semi-continuous from above (resp., upper semi-continuous from below), then there exists $x_{0} \in X$ such that $f\left(x_{0}\right)=\min _{x \in X} f(x)$ (resp., $f\left(x_{0}\right)=\max _{x \in X} f(x)$ ).

Proof. Assume that $f$ is lower semi-continuous from above on $X$. There exists a net $\left(y_{t}\right) \subset$ $C$ such that $f\left(y_{t^{\prime}}\right) \leq f\left(y_{t}\right)$ if $t^{\prime} \geq t$ and $f\left(y_{t}\right) \rightarrow \inf _{y \in C} f(y)$. Since $C$ is compact, without loss of generality, we may assume that $y_{t} \rightarrow y_{0}$. By the lower semi-continuity from above of $f(y)$, we have $f\left(y_{0}\right) \leq \lim _{t} f\left(y_{t}\right)$ and so $f\left(y_{0}\right)=\inf _{y \in C} f(y)$. The proof of upper semicontinuous from below case is similar and hence we omit the detail. This completes the proof.

Theorem 4.6 (von Neuman's minimax principle). Let $X$ and $Y$ be two nonempty compact convex subsets of topological vector spaces $E$ and $F$, respectively. Suppose that $f: X \times Y \rightarrow R$ is a real valued function satisfying the following conditions:

(1) $y \rightarrow f(x, y)$ is lower semi-continuous from above and quasi convex for each fixed $x \in X$, that is, $\{y: f(x, y)<r\}$ is convex for each $x \in X$;

(2) $x \rightarrow f(x, y)$ is upper semi-continuous from below and quasi concave for each fixed $y \in Y$, that is, $\{x: f(x, y)>r\}$ is convex for each $y \in Y$;

(3) for each $r \in R$, there exist $x_{i}, i=1,2, \ldots, n$, such that $A_{i}=\left\{y: f\left(x_{i}, y\right)>r\right\}$ is open and $Y=\cup_{i=1}^{n} A_{i}$;

(4) for each $r \in R$, there exist $y_{j}, j=1,2, \ldots, m$, such that $B_{j}=\left\{x: f\left(x, y_{j}\right)<r\right\}$ is open and $X=\cup_{j=1}^{m} B_{j}$.

Then $\max _{x \in X} \min _{y \in Y} f(x, y)=\min _{y \in Y} \max _{x \in X} f(x, y)$.

Proof. By the assumptions (1), (2) and Lemma 4.5, we know that $\max _{x \in X} \min _{y \in Y} f(x, y)$ and $\min _{y \in Y} \max _{x \in X} f(x, y)$ both exist. It is obviously that

$$
\max _{x \in X} \min _{y \in Y} f(x, y) \leq \min _{y \in Y} \max _{x \in X} f(x, y) .
$$

Now we show that

$$
\max _{x \in X} \min _{y \in Y} f(x, y)=\min _{y \in Y} \max _{x \in X} f(x, y) .
$$

If this is not true, then there would be a number $r \in R$ such that

$$
\max _{x \in X} \min _{y \in Y} f(x, y)<r<\min _{y \in Y} \max _{x \in X} f(x, y) .
$$

Define two maps $A, B: X \rightarrow 2^{Y}$ by $A x=\{y: f(x, y)>r\}$ and $B x=\{y: f(x, y)<r\}$ for $x \in X$. It is obvious that

$$
Y=\bigcup_{i=1}^{n} A x_{i}, \quad X=\bigcup_{j=1}^{m} B^{-1} y_{j} .
$$


It is direct to check that $A^{-1} y$ is convex for $y \in Y$ and $B x$ is convex for each $x \in X$ and, by Theorem 4.1, there exists $x_{0} \in X$ and $y_{0} \in Y$ such that $y_{0} \in A x_{0} \cap B x_{0} \neq \varnothing$. Hence we have $f\left(x_{0}, y_{0}\right)<r<f\left(x_{0}, y_{0}\right)$, which is a contradiction. This completes the proof.

Theorem 4.7 (Ky Fan's minimax inequality). Let $C$ be a compact convex subset of a topological vector space $X$. Let $f: C \times C \rightarrow R$ be a real valued function satisfying the following conditions:

(1) $\sup _{x \in C} f(x, y)$ is lower semi-continuous from above on $C$;

(2) $\left\{y: f(x, y) \leq \sup _{x \in C} f(x, x)\right\}$ is closed for each $x \in C$;

(3) $x \rightarrow f(x, y)$ is quasi-concave on $C$ for each $y \in C$.

Then $\min _{y \in C} \sup _{x \in C} f(x, y) \leq \sup _{x \in C} f(x, x)$.

Proof. By Lemma 4.5, we know that $\sup _{x \in C} f(x, y)$ obtains its minimum on $C$.

Now, we may assume that $\sup _{x \in C} f(x, x)=\mu<\infty$. Define a map $G: C \rightarrow 2^{C}$ by

$$
G(x)=\{y \in C: f(x, y) \leq \mu\}
$$

for all $x \in C$. The quasi-concavity of $x \rightarrow f(x, y)$ on $C$ for each $y \in C$ implies that $G$ is a KKM map. By the assumption (2), we know that $G(x)$ is compact. Therefore, it follows from Theorem 2.7 that $\cap_{x \in C} G(x) \neq \varnothing$, thus there exists $y_{0} \in C$ such that $y_{0} \in G(x)$ for all $x \in C$, that is, $f\left(x, y_{0}\right) \leq \mu$ for all $x \in C$. This immediately implies that

$$
\operatorname{minsup}_{y \in C} f(x, y) \leq \sup _{x \in C} f(x, x) .
$$

To end this paper, we give a function $f$ which satisfies all the conditions of Theorem 4.6.

Example 4.8. Let a function $f:[0,1] \times[0,1] \rightarrow R$ be defined by

$$
f(x, y)= \begin{cases}x+y & \text { if } y \in[0,1) \\ x+2 & \text { if } y=1\end{cases}
$$

Then we have

$$
\sup _{x \in[0,1]} f(x, y)= \begin{cases}1+y & \text { if } y \in[0,1) \\ 3 & \text { if } y=1\end{cases}
$$

Thus it follows that $\sup _{x \in[0,1]} f(x, y)$ is not lower semi-continuous, but lower semicontinuous from above. It is obvious that the set

$$
\left\{y: f(x, y) \leq \sup _{x \in[0,1]} f(x, x)=3\right\}=[0,1]
$$


is closed and

$$
\{x: f(x, 1)>r\}=\{x: x>r-2\}, \quad\{x: f(x, y)>r\}=\{x: x>r-y\}
$$

for all $y \in[0,1)$ are convex sets, that is, $x \rightarrow f(x, y)$ is quasi-concave on $C$ for each $y \in C$. Therefore, the function $f$ satisfies all the conditions of Theorem 4.6.

\section{Acknowledgments}

The authors are grateful to the referees for their valuable suggestions which help the revision of this paper. The second and fourth authors were supported by the Korea Research Foundation Grant (KRF-2000-DP0013).

\section{References}

[1] J.-P. Aubin and I. Ekeland, Applied Nonlinear Analysis, Pure and Applied Mathematics (New York), John Wiley \& Sons, New York, 1984.

[2] C. Bardaro and R. Ceppitelli, Some further generalizations of Knaster-Kuratowski-Mazurkiewicz theorem and minimax inequalities, Journal of Mathematical Analysis and Applications 132 (1988), no. 2, 484-490.

[3] F. E. Browder, The fixed point theory of multi-valued mappings in topological vector spaces, Mathematische Annalen 177 (1968), 283-301.

[4] S.-S. Chang and Y. Zhang, Generalized KKM theorem and variational inequalities, Journal of Mathematical Analysis and Applications 159 (1991), no. 1, 208-223.

[5] J. Dugundji and A. Granas, Fixed Point Theory. I, Monografie Matematyczne, vol. 61, Państwowe Wydawnictwo Naukowe (PWN), Warsaw, 1982.

[6] K. Fan, Fixed-point and minimax theorems in locally convex topological linear spaces, Proceedings of the National Academy of Sciences of the United States of America 38 (1952), 121-126.

[7] _ Sur un théorème minimax, Comptes Rendus Academie des Sciences Paris 259 (1964), 3925-3928 (French).

[8] Applications of a theorem concerning sets with convex sections, Mathematische Annalen 163 (1966), 189-203.

[9] __ Extensions of two fixed point theorems of F. E. Browder, Mathematische Zeitschrift 112 (1969), 234-240.

[10] A. Granas, KKM-maps and their applications to nonlinear problems, The Scottish Book (R. D. Mauldin, ed.), Birkhäuser, Massachusetts, 1981, pp. 45-61.

[11] A. Granas and J. Dugundji, Fixed Point Theory, Springer Monographs in Mathematics, Springer, New York, 2003.

[12] C. D. Horvath, Contractibility and generalized convexity, Journal of Mathematical Analysis and Applications 156 (1991), no. 2, 341-357.

[13] B. Knaster, C. Kuratowski, and S. Mazurkiewicz, Ein Beweis des Fixpunktsatzes fürn-dimensionale simplexe, Fundamenta Mathematicae 14 (1929), 132-137 (German).

[14] S. Park and H. Kim, Coincidence theorems for admissible multifunctions on generalized convex spaces, Journal of Mathematical Analysis and Applications 197 (1996), no. 1, 173-187.

[15] S. Singh, B. Watson, and P. Srivastava, Fixed Point Theory and Best Approximation: The KKMMap Principle, Mathematics and Its Applications, vol. 424, Kluwer Academic, Dordrecht, 1997.

[16] M. Sion, On general minimax theorems, Pacific Journal of Mathematics 8 (1958), 171-176.

[17] G. X.-Z. Yuan, KKM Theory and Applications in Nonlinear Analysis, Monographs and Textbooks in Pure and Applied Mathematics, vol. 218, Marcel Dekker, New York, 1999. 
[18] S. S. Zhang and G. S. Yang, Some further generalizations of Ky Fan's minimax inequality and its applications to variational inequalities, Applied Mathematics and Mechanics. Yingyong Shuxue he Lixue 11 (1990), no. 11, 961-968 (Chinese), Applied Mathematics and Mechanics (English ed.) 11 (1990), no. 11, 1027-1034.

Y. Q. Chen: Department of Mathematics, Foshan University, Foshan, Guangdong 528000, China E-mail address: yqchen@foshan.net

Y. J. Cho: Department of Mathematics Education and the RINS, College of Education, Gyeongsang National University, Chinju 660-701, Korea

E-mail address: yjcho@gsnu.ac.kr

J. K. Kim: Department of Mathematics Education, College of Education, Kyungnam University, Masan 631-701, Korea

E-mail address: jongkyuk@kyungnam.ac.kr

B. S. Lee: Department of Mathematics, Kyungsung University, Pusan 608-735, Korea

E-mail address: bslee@ks.ac.kr 